EPJ Web of Conferences 41, 05023 (2013)

DOI: 10.1051/epjconf/20134105023

(C) Owned by the authors, published by EDP Sciences, 2013

\title{
Intramolecular Charge Transfer Dynamics of a Planarized Analogue of 4-(dimethylamino)benzonitrile (DMABN) by Time-Resolved Fluorescence
}

\author{
Myeongkee Park ${ }^{1}$, So Young Kim ${ }^{1}$, D. $\mathrm{Im}^{2}$, Y. H. Rhee ${ }^{2}$, and Taiha Joo ${ }^{1}$ \\ ${ }^{1}$ Ultrafast dynamics Laboratory, Department of Chemistry, Pohang University of Science and \\ Technology (POSTECH), Pohang, 790-784, South Korea. \\ ${ }^{2}$ Synthetic Organic Chemistry Laboratory, Department of Chemistry, Pohang University of Science \\ and Technology (POSTECH), Pohang, 790-784, South Korea.
}

\begin{abstract}
Intramolecular charge transfer (ICT) of confined 1-tert-butyl-6-cyano-1, 2, 3, 4-tetrahydroquinoline (NTC6) is determined with a single time constant of $\sim 1$ ps regardless of solvents, although ICT of 4-(dimethylamino)benzonitrile (DMABN) shows dispersive dynamics in solvents.
\end{abstract}

\section{INTRODUCTION}

4-(dimethylamino)benzonitrile is well-known for its dual fluorescence; it emits mostly from the locally exited (LE) state at $350 \mathrm{~nm}$ in nonpolar solvents, whereas in polar solvents, such as acetonitrile, it exhibits much red-shifted intramolecular charge transfer (ICT) state fluorescence at around $470 \mathrm{~nm}$ [1]. Since Lippert's work [1], several models have been proposed such as twisted ICT (TICT) [2] and planar ICT (PICT) [3]. Initially, the TICT model, where fully twist of the dimethylamino group is required to undergo the ICT reaction, was widely accepted. However, recent advances on time-resolved techniques including femtosecond transient absorption (TA) and timeresolved fluorescence (TRF) have found evidences for somewhat complicated ICT reaction dynamics of DMABN [4,5]. In addition, our recent time-resolved fluorescence (TRF) and timeresolved fluorescence spectra (TRFS) of DMABN proposed a multi-faceted ICT process controlled in part by the conformational distribution of the ground state [6].

1-tert-butyl-6-cyano-1, 2, 3, 4-tetrahydroquinoline (NTC6), a planarized analogue of DMABN, also shows dual fluorescence and it has been considered to be an important evidence for the PICT model [3]. Theoretically, Gómez et al. showed that the PICT concept cannot be excluded in the ICT reaction [7], although a recent work by Hättig et al. indicated that the ICT reaction of the planarized NTC6 proceeds along the twisting coordinate because of the flexibility of the amino group to some extent [8].

To investigate the effect of molecular structures on ICT reaction, we employed highly time-resolved fluorescence measurements at several different wavelengths, and compared those of NTC6 and DMABN. In strong contrast to those of DMABN, TRFs of NTC6 do not exhibit the ultrafast rise (< $30 \mathrm{fs}$ ) components, and they display essentially the same ICT reaction rates regardless of the solvents used; acetonitrile and THF. So, the ICT dynamics of NTC6 is found to be homogeneous rather than the dispersive dynamics found in flexible DMABN.

This is an Open Access article distributed under the terms of the Creative Commons Attribution License 2.0, which permits unrestricted use, distribution, and reproduction in any medium, provided the original work is properly cited. 


\section{EXPERIMENTAL METHODS.}

A home-built Kerr lens mode-locked cavity-dumped Ti:sapphire laser was used as a light source. The excitation pulse at $276 \mathrm{~nm}$ was generated by using non-collinear sum frequency generation (SFG) of 830 and $415 \mathrm{~nm}$ pulses. TRF was measured by fluorescence upconversion method. Detailed description of the upconversion setup employing non-collinear SFG has been described elsewhere [9]. The instrument response function (IRF) was estimated to be 70 fs FWHM from difference frequency generation (DFG) of the scattered pump pulses $(276 \mathrm{~nm})$ and gate pulses (830 $\mathrm{nm})$. At longer wavelength detection, time-resolution is estimated to be better because of the smaller group velocity mismatch (GVM) between the fluorescence and the gate pulse.

\section{RESULTS AND DISCUSSIONS}

In acetonitrile, ultrafast decay and rise of DMABN consisting of the very fast rise ( $<30 \mathrm{fs}), 180 \mathrm{fs}$, and 2.7 ps are observed (Fig. 1a). TRFs of NTC6 indicate that ICT of NTC6 occurs faster than DMABN and measured ICT reaction rates are about 1.1 ps- 1 and $350 \mathrm{fs}-1$. However, in the TRFs of NTC6 there is no instantaneous rise, which indicates barrierless reaction (Fig 1c). This phenomenon supports possibility of faster ICT reaction rate for a certain geometry in a pre-twisted structure [8], and this channel is not allowed for NTC6 because of its confined structure.

TRFs of DMABN and NTC6 dissolved in moderately polar THF shown on figure 2 are quite different. Time profiles of DMABN show decay and rise times of 13 ps which are much longer than the 2.7 ps of DMABN in acetonitrile (Fig. 2a). However TRFs of NTC6 dissolved in THF show lifetimes of $1.4 \mathrm{ps}$ similar to that of NTC6 in acetonitrile. These solvent dependent ICT rates for DMABN and the absence of the solvent dependence for NTC6 may be originated from the continuous increase of the twist angle of the amino group along the solvent coordinate, like some molecules conforming to the Sumi/Marcus electron transfer $[10,11]$ model. This model, of course, cannot be applied to NTC6 that has a fixed twist angle. Therefore, NTC6 may show the homogeneous ICT reaction rather than multifaceted ICT reactions of DMABN.
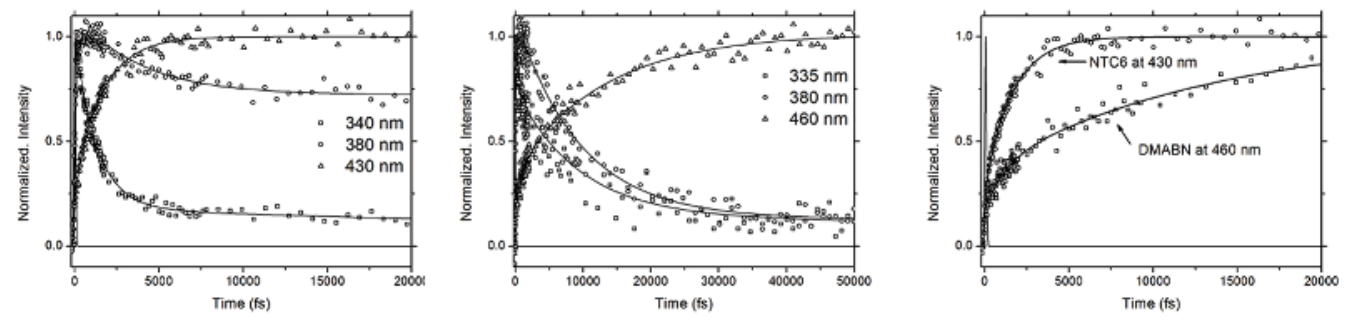

Fig. 1. Time profiles of THF solutions. (a) NTC6, (b) DMABN, and (c) Comparison on time profiles on ICT states of NTC6 and DMABN
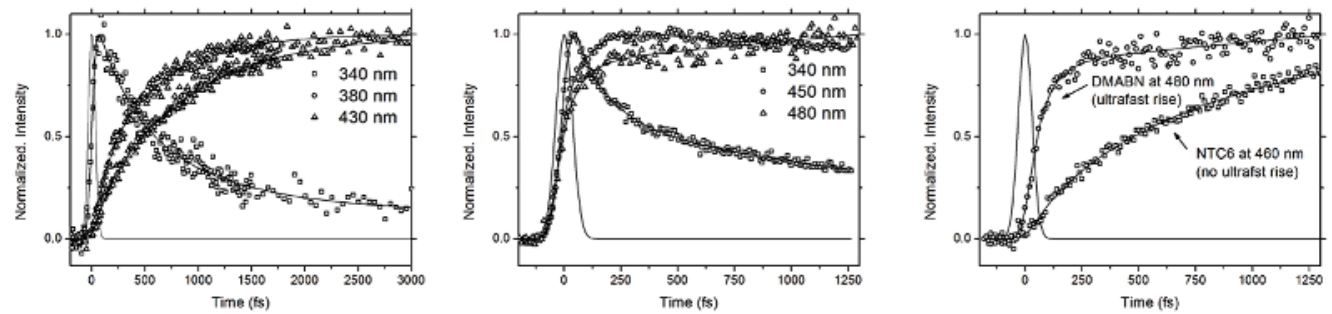

Fig. 2. Time profiles of acetonitrile solutions. (a) NTC6, (b) DMABN, and (c) Comparison on time profiles on ICT states of NTC6 and DMABN. Only DMABN has a ultrafast rise ( $<30 \mathrm{fs})$ 


\section{CONCLUSIONS}

We compared ultrafast ICT dynamics of DMABN and NTC6 by using highly time-resolved fluorescence. NTC6 shows homogeneous ICT reaction rate of about 1 ps-1 regardless of solvent polarities, whereas DMABN exhibits strong solvent dependence and dispersive reaction rates including instantaneous reaction. Our results strongly support unified ICT models consisting of TICT, PICT [6].

\section{References}

[1] E. Lippert, W. Lüder, and H. Boss, Advances in Molecular Spectroscopy (Pergamon Press, Oxford, 1962).

[2] W. Rettig, Ang. Chem. Int. Ed. 25, 971 (1986).

[3] K. A. Zachariasse, S. I. Druzhinin, W. Bosch, and R. Machinek, J. Am. Chem. Soc. 126, 1705 (2004).

[4] A. Pigliucci, E. Vauthey, and W. Rettig, Chem. Phys. Lett. 469, 115 (2009).

[5] T. Gustavsson, P. B. Coto, L. Serrano-Andés, T. fujiwara, and E. C. Lim, J. Chem. Phys. 131, 31101 (2009).

[6] M. Park, C. H. Kim, and T. Joo, "Multifaceted Ultrafast Intramolecular Charge Transfer (ICT) reaction of 4-(dimethylamino)benzonitrile (DMABN)" (to be submitted).

[7] I. Gomez, M. Reguero, and M. Boggio-Pasqua, M. A. Robb, J. Am. Chem. Soc. 127, 7119 (2005).

[8] C. Hättig, A. Hellweg, and A. Köhn, J. Am. Chem. Soc. 128, 15672 (2006).

[9] H. Rhee and T. Joo, Opt. Lett. 30, 96 (2005).

[10] H. Sumi and R. A. Marcus, Chem. Phys. 84, 4894 (1986).

[11] M. Liu, N. Ito, M. Maroncelli, D. H. Waldeck, A. M. Oliver, and M. N. Paddon-Row J. Am. Chem. Soc. 127, 17867 (2005). 